PROC. OF JSCE,

No. 203, JULY 1972

\title{
ON THE DYNAMIC CHARACTERISTICS OF ASPHALT MIXTURES
}

\author{
By Tatsuo OHMACHI ${ }^{*}$ and Takashi Watanabe**
}

\section{SYNOPSIS}

Dynamical properties of asphalt mixtures were studied by vibrating the cantilever beams of the mixtures. We found that the properties of actual mixture are so complicated that we cannot approximate by the simple linear viscoelastic models such as Voigt type and Maxwell type. When the vector locus of complex elastic modulus $E^{*}(i \omega)$ for asphalt mixtures was plotted with the dynamic elastic modulus as abscissa and loss modulus as ordinate, it forms a wing shape locus and is not so simple as the semicircle (Maxwell) or straight half line (Voigt).

We proposed a new index which shows the mechanical properties of mixtures. It was defined by the length between the intersection of two asymptotes and frequency characteristic curve of mixture on $E^{*}(i \omega)$ and frequency plane at which the phase difference between stress and strain was $45^{\circ}$. From the vertical location of both ends of the line, we can estimate the resistance to deformation of mixtures as a function of frequency, since the top end shows the elastic characteristic at infinitive frequency and the bottom end shows the actual stiffness of the mixture. The horizontal location of the line shows the frequency at which the phase difference between stress and strain is $45^{\circ}$, when the viscous and elastic resistance is equal at this frequency. The length of the line shows the rate of change from viscous to elastic state when frequency increases, for example, the longer the length, the more gradual the change will be.

Some of the test results are discussed, applying the characteristic line concept, and we found that the thin asphalt film with less than about 5 micron

* Post Graduate Student, Civil Eng. Dep't. Tokyo Univ.

** Professor, Civil Eng. Dep’t. Tokyo Institute of Technology thickness has different properties from thicker ones. And also the mixing effect of mineral particles to asphalt are discussed.

\section{INTRODUCTION}

One of the important components of flexible pavements is the asphalt mixtures. Many works have been carried out to define the mechanical properties of mixtures, however, the results obtained until now are not so satisfactory with regard to the complicated characteristics of mixtures. For example, mathematical expression of the characteristics by linear viscoelastic theory such as the combination of elastic springs and viscous dashpots, leads not only to very complicated analysis but to difficulties in understanding the physical meanings of the results. And the stiffness concept proposed by Van der Poel ${ }^{1)}$ and modified by Heukelom and Klomp2) does not imply the time lag between stress and strain induced in the material so that it may lead us to misconception of the real characteristics of the viscoelastic asphalt mixtures.

Since the road surface is subjected to very varying conditions such as low to high temperature, slow to rapid moving vehicle, parameters of indices for mechanical properties of asphalt mixtures should satisfy the following conditions:

1. There should be little or no difficulty to find the difference of properties between various types of mixtures;

2. They should be applicable over a wide range of temperature and loading periods;

3. They should be useful in design problems and in simple forms of expression.

We have carried out experiments to determine these indices and some of the results on this problem are reported here.

\section{EXPERIMENTS}

Vertical cantilever beam specimen fixed on the 


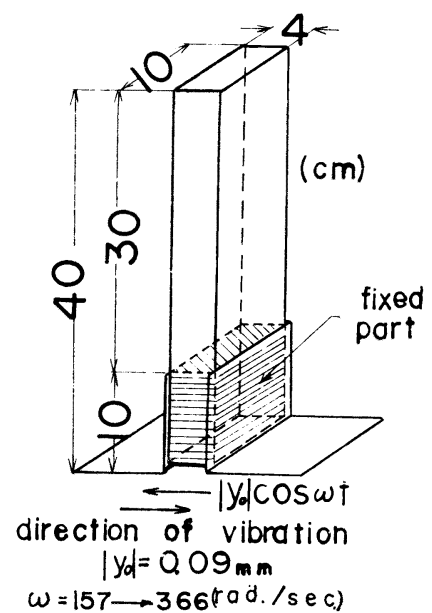

Fig. 1 Schematic explanation of experiments.

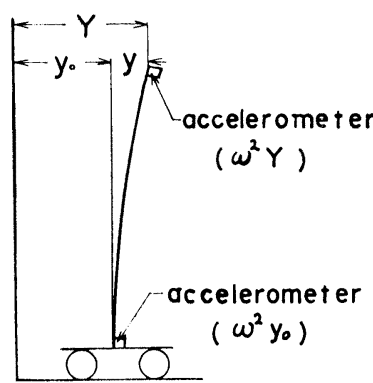

Fig. 2 Coordinate system.

vibrating table at one end was oscillated horizontally by sinusoidal wave, as shown in Fig. 1 . Accelerations at top and bottom of the beam were measured by accelerometers and a syncroscope as shown in Fig. 2. The amplitude of the vibrating table had been controlled to be below $0.09 \mathrm{~mm}$ which gave a maximum strain of $4 \times 10^{-4}$ to the specimen so that the linear viscoelastic properties could be assumed in these experiments.

The experiments were conducted at a temperature between $0^{\circ} \mathrm{C}$ to $45^{\circ} \mathrm{C}$ and a frequency range of $25 \mathrm{cps}$ to $58.3 \mathrm{cps}$ was used. The cantilever specimen had a length of $40 \mathrm{~cm}$, a width of $10 \mathrm{~cm}$ and a depth of about $4 \mathrm{~cm}$. The width and depth of the specimen were measured at three points and the averages of three measured values were used for the analysis of the experimental results. These measurements showed that every specimen was of uniform size and the difference in size was less than $6 \%$. The top $30 \mathrm{~cm}$ of the specimen was subjected to vibratioan and the lower $10 \mathrm{~cm}$ was clamped with steel plates to the vibrating table as shown in Fig. 1.

\section{CALCULATION OF COMPLEX MODULUS OF MIXTURES}

In this type of experiments, the response of the beam will be sinusoidal. However, the strain and stress will not be in the same phase due to energy dissipation. The stress can be shown by two components vectorially as shown in Fig. 3, one in same phase with the strain and the other $90^{\circ}$ from the strain. Thus, stress and strain relationship can be written by using the complex modulus as shown in Eq. (1),

$$
\sigma(t)=E^{*}(i \omega) \varepsilon(t)
$$

and

$$
E^{*}(i \omega)=E^{\prime}(\omega)+i E^{\prime \prime}(\omega)
$$

From Eq. (1) and (2), we have

$$
E^{*}(i \omega)=\left|E^{*}\right| e^{i \delta}
$$

where

$$
\begin{aligned}
& i=\sqrt{-1} \\
& \sigma(t)=\text { stress as a function of time }\left(\mathrm{kg} / \mathrm{cm}^{2}\right), \\
& \varepsilon(t)=\text { strain as a function of time }(-), \\
& E^{*}(i \omega)=\text { complex modulus which is a func- } \\
& \text { tion of frequency, } \\
& E^{\prime}(\omega)=\text { real part of complex modulus, which } \\
& \text { is often called storage modulus }\left(\mathrm{kg} / \mathrm{cm}^{2}\right), \\
& E^{\prime \prime}(\omega)=\text { imaginary part of complex modulus, } \\
& \text { which is often called loss modulus } \\
& \left(\mathrm{kg} / \mathrm{cm}^{2}\right) \text {, } \\
& \left|E^{*}\right|=\text { absolute value of complex modulus, } \\
& \text { which is often called dynamic (elastic) } \\
& \text { modulus }\left(\mathrm{kg} / \mathrm{cm}^{2}\right) \text {, } \\
& \delta=\text { argument of complex modulus (rad.) }
\end{aligned}
$$

If we take the coordinates as shown in Fig. 2, the equation for this vibrating system is

$$
\frac{\gamma A}{g} \frac{\partial^{2} y}{\partial t^{2}}+\eta I \frac{\partial^{5} y}{\partial t \partial x^{4}}+E^{\prime} I \frac{\partial^{4} y}{\partial x^{4}}=-\frac{r A}{g} \frac{\partial^{2} y_{0}}{\partial t^{2}}
$$

where

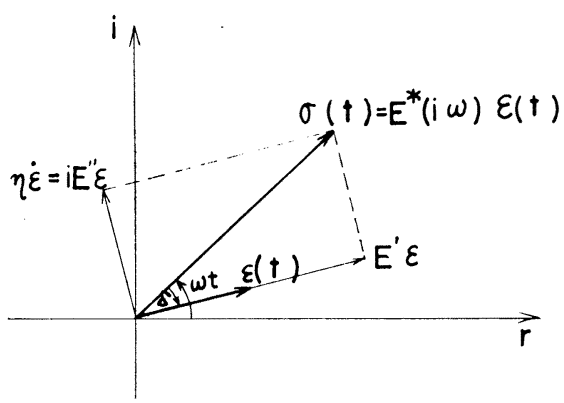

Fig. 3 Vectorial phase of stress components and strain. 
$\gamma=$ unit weight $\left(\mathrm{kg} / \mathrm{cm}^{3}\right)$,

$A=$ cross-sectional area $\left(\mathrm{cm}^{2}\right)$,

$I=$ moment of inertia $\left(\mathrm{cm}^{4}\right)$,

$y=$ relative displacement of the beam $(\mathrm{cm})$,

$y_{0}=$ absolute displacement of the table $(\mathrm{cm})$,

$\eta=$ coefficient of viscosity $\left(\mathrm{kg} / \mathrm{cm}^{2} / \mathrm{sec}\right)$.

The applied force, i.e. the right hand side of Eq. (4), due to the sinusoidal motion of the exciting table, can be given by

$$
y_{0}=\left|y_{0}\right| \cos \omega t
$$

Neglecting the effect of higher modes of vibration, the relative displacement of the free end $y(t)$ can be obtained by the solution of Eq. (4)

$$
y(t)=\frac{1.57\left|y_{0}\right| \frac{\omega^{2}}{p^{2}}}{\sqrt{\left(1-\frac{\omega^{2}}{p^{2}}\right)^{2}+\tan ^{2} \delta}} \cos (\omega t-\theta) \cdots \cdots(
$$

where

$$
\tan \theta=\frac{\tan \delta}{1-\frac{\omega^{2}}{p^{2}}}, \quad p=(\beta l)^{2} \sqrt{\frac{E^{\prime} I g}{\gamma A l^{4}}} \cdots \cdots
$$

$l=$ length of the beam $(\mathrm{cm})$,

$\beta l=1.875$ for the first mode of vibration.

In our experiments, the ratio of amplitude, $F$ $=|y| /\left|y_{0}\right|$, the phase difference $\theta$ and the frequency $\omega$ were measured by using accelerometers attached to the top of the specimen and the vibrating table, as shown in Fig. 2. From these measured values, the complex modulus $E^{*}(i \omega)$ of the mixtures is calculated by the following equations which are easily derived from Eq. (6) and (7):

$$
\begin{aligned}
& \tan \delta=\frac{1.57 \sin \theta}{F+1.57 \cos \theta} \\
& E^{\prime}=\left|E^{*}\right| \cos \delta=\frac{1}{\beta^{4}} \frac{\gamma A}{g I}\left(1+\frac{1.57}{F} \cos \theta\right) \omega^{2} \\
& \quad=1.67 \frac{W}{I}\left(1+\frac{1.57}{F} \cos \theta\right) \omega^{2}
\end{aligned}
$$

where

$$
W=\text { total weight of specimen }(\mathrm{kg}) .
$$

Though any two of the terms in $E^{\prime}$, $E^{\prime \prime},\left|E^{*}\right|$ and $\delta$ can completely define the complex modulus at a given temperature and frequency, we chose the dynamic modulus $\left|E^{*}\right|$ and phase difference $\delta . \quad\left|E^{*}\right|$ is also defined as the

\begin{tabular}{|c|c|c|c|c|c|}
\hline \multicolumn{2}{|c|}{ Asphalt content } & $\begin{array}{l}\text { Code } \\
\text { Letter }\end{array}$ & $\begin{array}{c}\text { Asphalt } \\
(\text { Pen. 80/100) }\end{array}$ & $\begin{array}{l}\text { Code } \\
\text { Letter }\end{array}$ & $\begin{array}{c}\text { Asphalt } \\
\text { (Pen. 20/40) }\end{array}$ \\
\hline $\begin{array}{l}\text { Filler } \\
\left(\begin{array}{l}\mathrm{CaCO} \\
\text { powder }\end{array}\right)\end{array}$ & $\frac{V_{A S}}{V_{F}}$ & $F$ & $\begin{array}{l}40,50,60 \\
70,100(\%)\end{array}$ & $f$ & $30,40,60)$ \\
\hline $\begin{array}{l}\text { Sand } \\
\left(\begin{array}{l}0.3 \sim \\
0.6 \mathrm{~mm}\end{array}\right)\end{array}$ & $\frac{V_{A s}}{V_{S}}$ & $S$ & $\begin{array}{l}10,20,30 \\
40,60(\%)\end{array}$ & $s$ & $10,20,40$ \\
\hline $\begin{array}{l}\text { Gravel } \\
\left(\begin{array}{c}2.5 \sim \\
5 \mathrm{~mm}\end{array}\right)\end{array}$ & $\frac{V_{A s}}{V_{G}}$ & $G$ & ${ }_{43}^{11}, 18,25$ & $g$ & $11,18, \underset{43}{25})$ \\
\hline
\end{tabular}
ratio of the amplitude of stress and strain, and $\delta$ is the phase difference between stress and strain.

\section{EXPERIMENTAL RESULTS}

The rheological properties of asphalt
Table 1 Asphalt contents of mineral particles

mixtures and the influences of mixture components on them were investigated by the specimens listed in Table 1. For each type of specimens, more than three samples were prepared and tested under identical condition. Dynamic modulus $\left|E^{*}\right|$ and phase difference $\delta$ were calculated by Eq. ( $8 \mathrm{a})$, $(8 \mathrm{~b})$ for each tested frequency, and the frequency characteristics of these values can obtained by averaging the values of three identical samples.

In the following analysis, the results obtained from F70 specimen which consists of mineral filler and asphalt (Pan. 80/100), with the asphalt content in volume $V_{A S} / V_{F}=70 \%$, will be shown as an example.

(1) Relationship between Time and Temperature

Fig. 4 shows the relationship between dynamic modulus and circular frequency. It can be noticed that time and temperature have an approximately equivalent effect on the viscoelastic response of asphalt mixtures, that is, the dynamic modulus increases either with the increase in frequency or decrease in temperature. At the same time, it can be seen also that the asphalt mixtures used in this study are thermorheologically simple materials since all the dynamic modulus curves

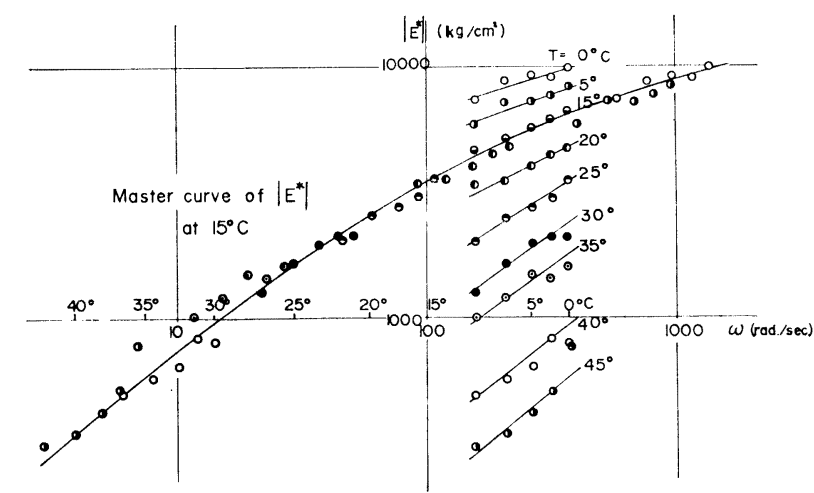

Fig. 4 Derivation of master curve for $\left|E^{*}\right|$ from the experimental results (ref. temp. $15^{\circ} \mathrm{C}$, sample; F70). 
can be brought to form a smooth master curve by horizontal shifts without changing each shape. A reference temperature, $T_{0}$, equal to $15^{\circ} \mathrm{C}$, was arbitrarily chosen.

Horizontal distances between each pair of adjacent curves of dynamic modulus were measured with the result as shown in Fig. 4 and also by the phase difference and frequency curve. Averaging these two distances, the horizontal shift factor, $\log a_{T}$, was evaluated. The adjustment for the density difference due to temperature change suggested by the kinetic theory was disregarded, since the shift of vertical position due to this adjustment may be small and negligible.

Fig. 4 also shows a master curve of $\left|E^{*}\right|$ in which the reference temperature and frequency are $15^{\circ} \mathrm{C}$ and $\omega=100 \mathrm{radian} / \mathrm{sec}$. It is obtained by shifting horizontally the experimental results at different temperatures, it gives also the frequency shifts $\log a_{T}$, on the horizontal axis of $\left|E^{*}\right|=$ $1000 \mathrm{~kg} / \mathrm{cm}^{2}$ as shown in the figure. Hence, we can easily obtain a master curve of $\left|E^{*}\right|$ at any arbitrary temperature between $40^{\circ} \mathrm{C}$ and $0^{\circ} \mathrm{C}$ by shifting horizontally the reference point of vertical axis, i.e., $\omega=100$ ( $\mathrm{rad} . / \mathrm{sec})$, to the corresponding temperature.

\section{(2) The Influence of Temperature on Shift}

Factor, $a_{T}$

The shift factor evaluated for all the specimens

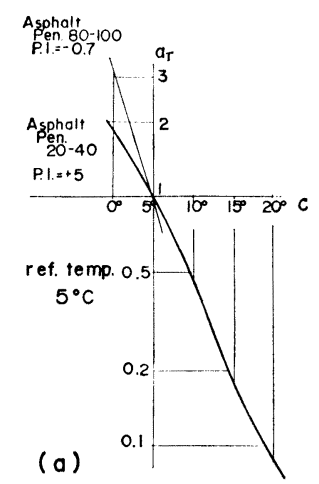

Fig. 5 Relations between shift factor $a_{T}$ and temperature.
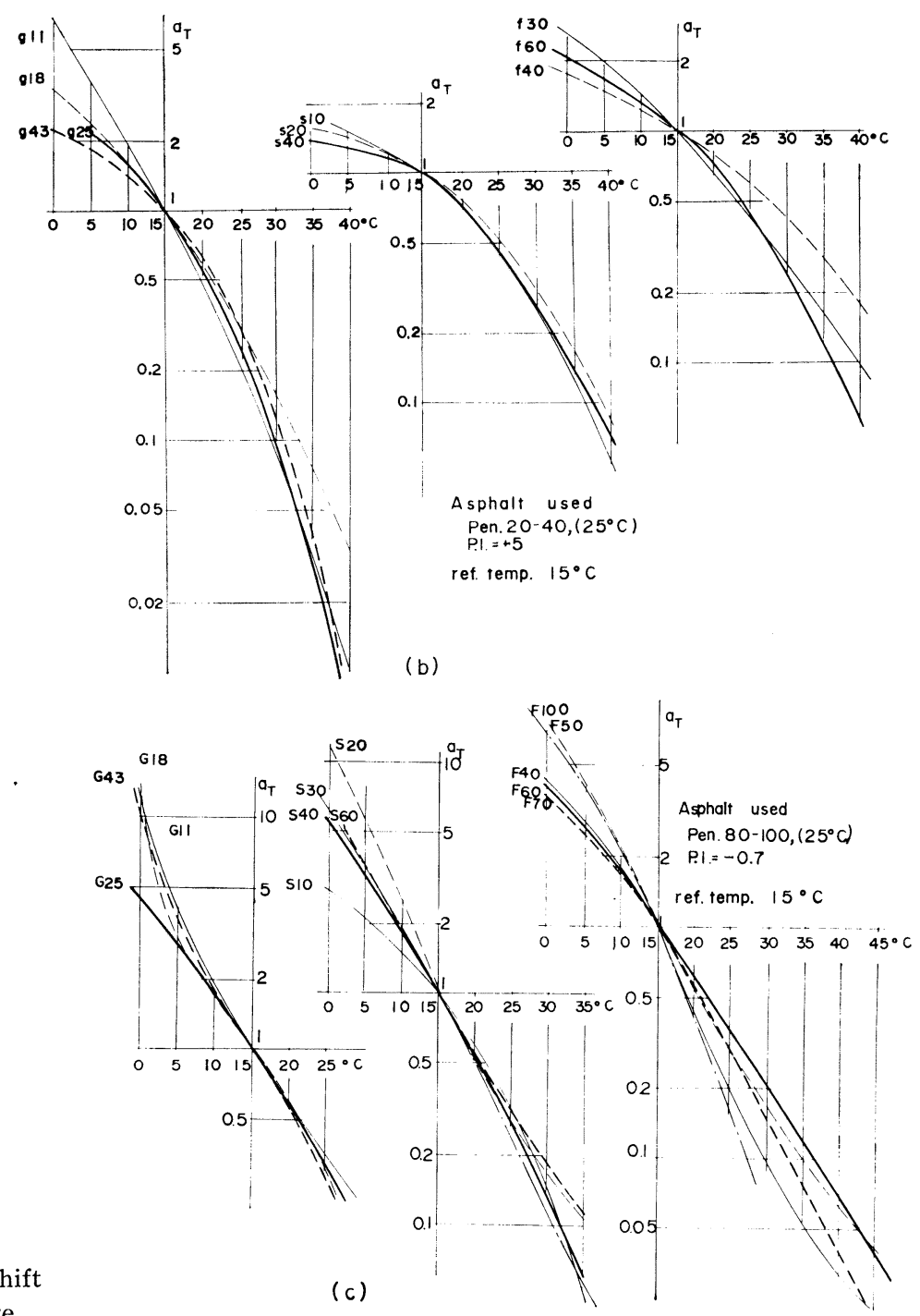
are given in Fig. 5(a), (b), (c) as a function of temperature. As for shift factor, Williams, Landels and Ferry3) showed that for most of the high polymer materials the relationship can be written as

$$
\log a_{T}=\frac{-C_{1}^{0} \cdot\left(T-T_{0}\right)}{C_{2}{ }^{0}+\left(T-T_{0}\right)}
$$

where

$T_{0}=$ arbitrarily chosen reference temperature,

$C_{1}{ }^{0}, C_{2}{ }^{0}=$ material constants for $T_{0}$

$\left(C_{1}{ }^{0}>0, C_{2}{ }^{0}>0\right)$.

If we transform the above equation into

$$
\left(\log a_{T}+C_{1}{ }^{0}\right)\left\{\left(T-T_{0}\right)+C_{2}{ }^{0}\right\}=C_{1}{ }^{0} C_{2}{ }^{0}
$$

we note that Eq. (9) gives a hyperbola when $\log a_{T}$ is plotted against $\left(T-T_{0}\right)$ as in Fig. 6. However, Fig. 5 indicates that almost all the curves obtained do not resemble those of hyperbola. It means that the asphalt mixtures do not have characteristics as simple as the normal high polymers.

The binder content of the mixture did not influence the value of $a_{T}$, so much as seen in Fig. 5. However, shift factor $a_{T}$ depended more on the type of asphalt used. Krokosky and Chen ${ }^{4)}$ pointed out similar tendency with regard to shift factor $a_{T}$, by compression stress relaxation tests.

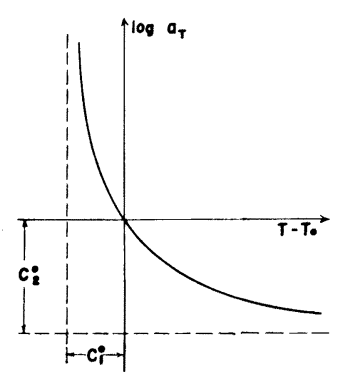

Fig. 6 W. L.F. equation

\section{(3) Difference from the Vibrating Systems with Constant Coefficients}

The experimental data of F70 specimen are shown in Fig. 7 (a), (b) in terms of relative amplitude radio $F$, i.e., $|y| /\left|y_{0}\right|$, and phase difference $\theta$. Both curves in Fig. 7 appear to be similar to those of forced vibration with viscous damping, as shown in Fig. 8(a), (b), (c). However, there exists a very important difference between them. To examine the vibrating characteristics of asphalt mixtures in detail, Fig. 7 and Fig. 8 are redrawn on a polar cooreinates with the relative amplitude radio $F$ as the radius and the phase difference $\theta$ as the angle, as shown in Fig. 9.
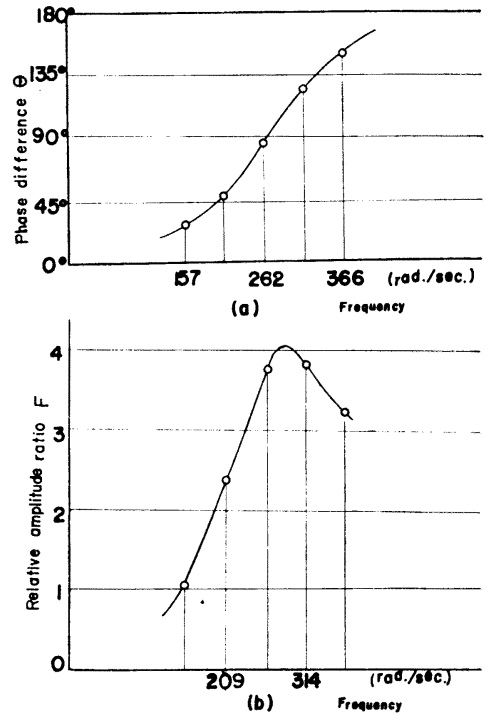

Fig. 7 Relative amplitude ratio $F$ and phase difference $\theta$ for F70 at $15^{\circ} \mathrm{C}$.
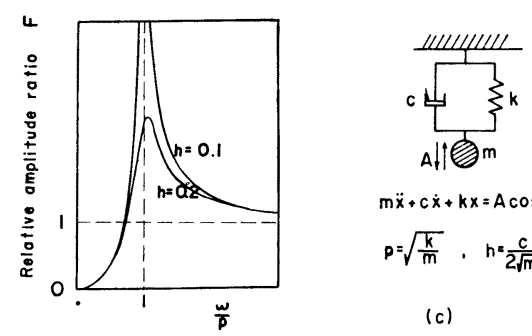

$m \ddot{x}+c \dot{x}+k x=A \cos \omega t$ $p=\sqrt{\frac{k}{m}}, h=\frac{c}{2 \sqrt{m k}}$

(c)

(b)

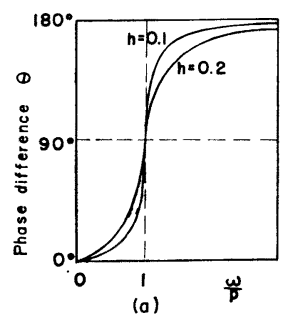

Fig. 8 Relative amplitude ratio $F$ and phase difference $\theta$ for one-degree system.

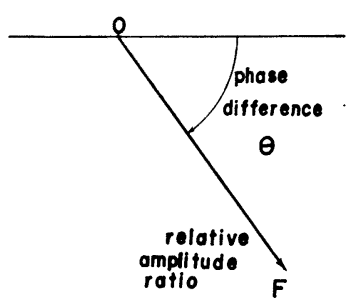

Fig. 9 Vectorial representation of $F$ and $\theta$. 


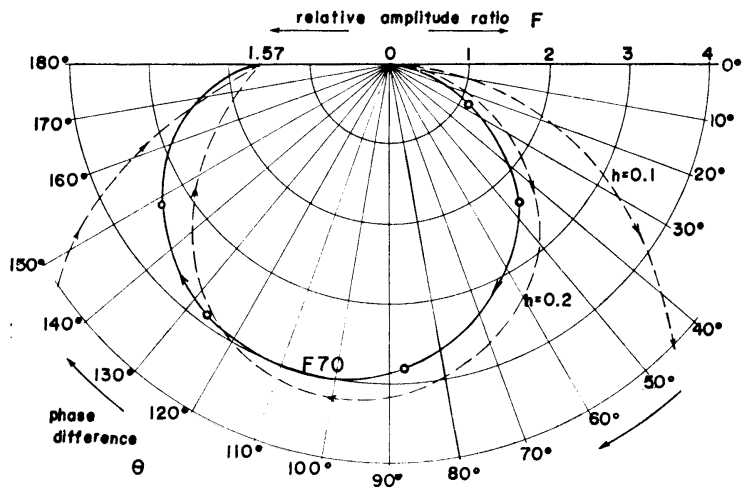

Fig. 10 Vector locus for second-order of vibration system $(h=0.1, h=0.2)$ and for F70.

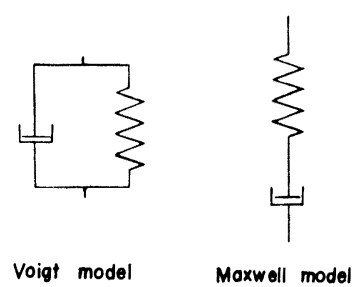

Fig. 11 Voigt model and Maxwell model.
This kind of representation is referred to as vector locus for vibrating system. The solid line in Fig. 10 shows the vector lucus for F70, and the dotted ones for the constant coefficient systems (in Fig. 8) with the damping factor $h$ $=0.1$ and $h=0.2$ respectively. The end of the vector moves in the dirrection of the arrow with the increase in frequency. It is important that the solid line deviates gradually from the dotted curve of high damping system and converges to the low damping, in other words, the asphalt mixture has a large damping factor in the range of lower frequency while in the range of higher frequency damping factor is low at the temperature of $15^{\circ} \mathrm{C}$.

In general, viscoelastic bodies are often analysed and simulated in terms of a Voigt model or a Maxwell model, shown in Fig. 11, due to the simplicity in analysis. It would be expected from the above discussion that this idealization with simple models is applicable in very limited degree. To illustrate the difference from these viscoelastic models, the master curves for F70 are shown in Fig. 12 again. Here both the master curves of phase difference $\delta$ and dynamic modulus $\left|E^{*}\right|$ are shown. In Fig. 13, complex modulus are plotted on a polar coordinate with the dynamic modulus $\left|E^{*}\right|$ as the radius and the phase difference $\delta$ as the angle. The end of the vector $E^{*}(i \omega)$ moves in the direction of the arrow when the frequency increases so that the vector locus for asphalt mixture forms a wing like shape. On the

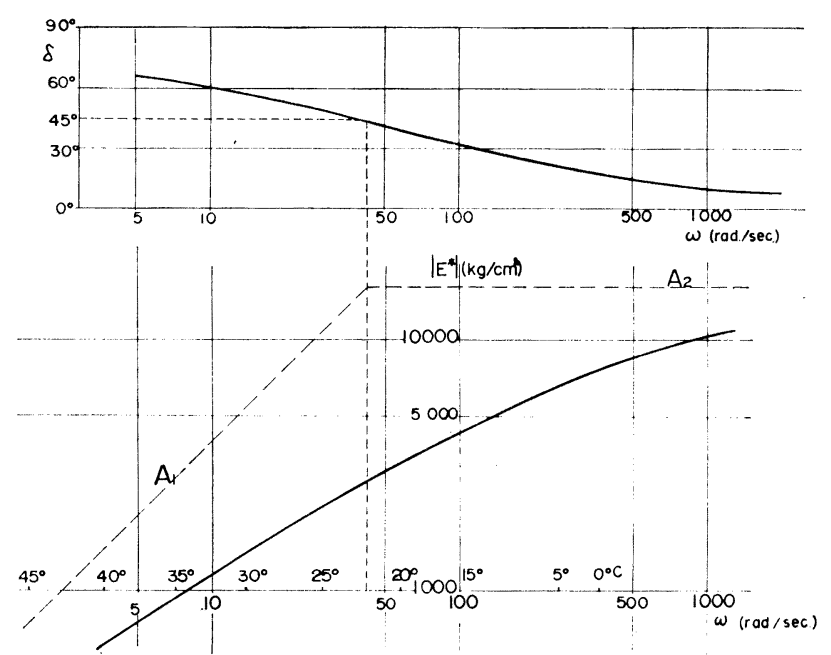

Fig. 12 Master curves of $\left|E^{*}\right|$ and $\delta$ at $15^{\circ} \mathrm{C}$ for and sample F70.

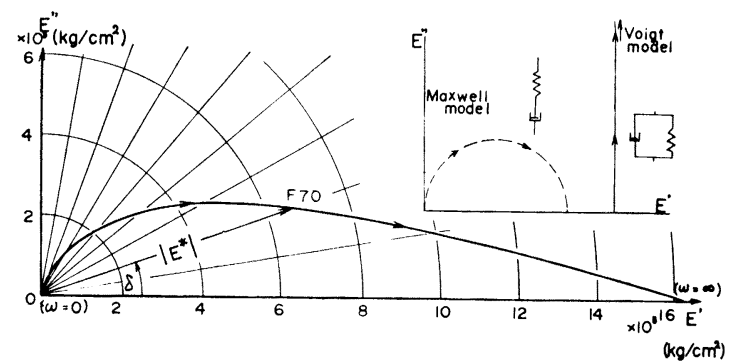

Fig. 13 Vector loci of $E^{*}$ for F70, Voigt model and Maxwell model.

other hand, the vector locus for a Maxwell model forms a semicircle, and for a Voigt model forms a half line. In Fig. 13 it may be observed that for asphalt mixtures and a Maxwell model phase difference $\delta$ approches $90^{\circ}$ when $\omega$ approaches 
zero and $\delta$ approaches $0^{\circ}$ when $\omega$ tends to infinitive, while the reverse occures for a Voigt model. Fig. 13 shows that we have to change the coefficients of springs and dash pots successively in order to represent the behavior of asphalt mixtures exactly, when the combination of such variety of models as Hookian springs and Newtonian dash pots are chosen.

\section{(4) Viscoelastic Properties of Asphalt Mixtures}

In general, elastic response is in same phase between stress and strain for $\delta=0^{\circ}$, and viscous response has a phase lag of $\pi / 2$ radian between stress and strain for $\delta=90^{\circ}$.

According to Fig. 12, the mechanical properties of asphalt mixtures varies gradually from viscous to elastic state with the increase in frequency. Two dotted lines $A_{1}$ and $A_{2}$ are the asymptotes corresponding to $\omega \rightarrow 0$ and $\omega \rightarrow \infty$ respectively.

A master curve of $\delta$ in Fig. 12 states that $\delta$ approaches $90^{\circ}$ when $\omega$ approaches $0(\mathrm{rad} / \mathrm{sec})$ so that asphalt mixture can be considered as a viscous body. Since stress and strain can be expressed by the sinusoidal function of time, the stress and strain relationship can be written as

$$
\sigma(t)=\eta_{0} \frac{d \varepsilon(t)}{d t}=i \omega \eta_{0} \varepsilon(t)
$$

where

$$
\eta_{0}=\text { coefficient } \text { of viscosity when } \omega \rightarrow 0
$$$$
\left(\mathrm{kg} / \mathrm{cm}^{2} / \mathrm{sec}\right) \text {. }
$$

substitution in Eq. (1) gives

$$
\left|E^{*}\right| \rightarrow \omega \eta_{0}
$$

then

$$
\log \left|E^{*}\right| \rightarrow \log \omega+\log \eta_{0}
$$

Therefore, a line $A_{1}$ having a slope of $45^{\circ}$ to the abscissa on a logarithmic graph is obtained as an asymptote.

On the other hand, it can also be observed in Fig. 12 that $\delta$ approaches $0^{\circ}$ when $\omega$ tends to infinitive so that, in this case, this material can be considered as an elastic body. Then, $\left|E^{*}\right|$ becomes independent of frequency and approaches the constant value of $E_{\infty}$. Therefore, a horizontal line $A_{2}$ is obtained as another asymptote.

It is interesting that the phase difference $\delta$ is $45^{\circ}$ at the frequency where the two asymptotes intersect. When the phase difference $\delta$ is equal to $45^{\circ}$, the resistances to deformation produced by the elastic and viscous component should be equal. The same condition can be defined by the loss tangent as

$$
\tan \delta=\frac{E^{\prime \prime}}{E^{\prime}}=1
$$

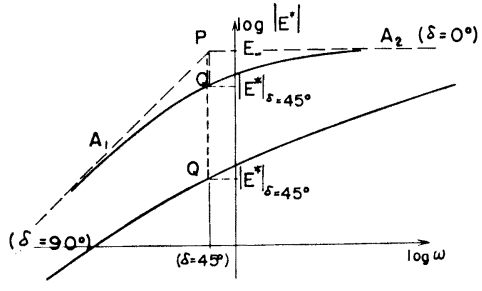

Fig. 14 Relations between $\left|E^{*}\right|$ and their asymptotes.

We chose the point at $\delta$ equal to $45^{\circ}$ as a reference point to compare the viscoealstic properties of asphalt mixtures. In Fig. 14, the point $P$ shows the frequency or temperature at which the phase difference $\delta$ is equal to $45^{\circ}$, and it is on the intersection of the two asymptotes $A_{1}, A_{2}$. Then we can express the frequency or temperature at $\delta=45^{\circ}$ and two asymtotes by a point $P$.

The actual experimental results were shown as the curves in Fig. 14. The curves show that the change of properties from viscous to elastic state will take place gradually when the frequency increases. If the material has the frequency characteristic expressed by two asymptotes $A_{1}, A_{2}$, it means that it is completely viscous below the frequency at $P$ and also perfectly elastic over the point $P$. However, the actual curve showed the deviation from these asymptotes, as shown in the figure, which means that the actual property is a combination of the properties of elastic and viscous state. Therefore the length of line $P Q$ shows the degree of deviation from pure viscous or elastic material. The point $Q$ on the curve can be obtained just below the point $P$ in the figure and it shows the point where the actual material shows $\delta=45^{\circ}$. We call this line $P Q$ as the characteristic line of a mixture.

The length of characteristic line $P Q$ shows the value of $\log \left(E_{\infty} /\left|E^{*}\right|_{\delta=45^{\circ}}\right)$. The larger the length of $P Q$, the more gradual is the change of properties from viscous to elastic state due to frequency increase.

From the discussion above, it is obvious that the vertical position of $P$ can only be detemined by the stiffness for $\omega \rightarrow \infty$, i.e., $E_{\infty}$. For example, if the material is very stiff at high frequency, the vertical position of $P$ is high. On the other hand, the horizontal location of the characteristic line is determined by the frequency at $\delta=45^{\circ}$ which depends upon the ratio of $\eta_{0} / E_{\infty}$. The relations between the location of line $P Q$ and the viscoelastic properties of the material are shown in Table 2 .

It should also be important to obtain the 
Table 2 Relations between the line $P Q$ and the characteristics of the mixture.

\begin{tabular}{l|c|c}
\hline \multicolumn{3}{c}{ Vertical Location of $P$} \\
\hline $\begin{array}{c}\text { elastic state } \\
\left(\delta \rightarrow 0^{\circ}\right)\end{array}$ & $\begin{array}{c}\text { change due to } \\
\text { increase in } E_{\infty}\end{array}$ & $\begin{array}{c}\text { change due to } \\
\text { decrease in } E_{\infty}\end{array}$ \\
\hline & Vertical Location of $Q$ & $\downarrow$ \\
\hline $\begin{array}{c}\text { elastic }=\text { viscous } \\
\left(\delta=45^{\circ}\right)\end{array}$ & increase in $\left|E^{*}\right|_{\delta=45^{\circ}}$ & decrease in $\left|E^{*}\right|_{\delta=45^{\circ}}$ \\
\hline & $\uparrow$ & $\downarrow$ \\
\hline
\end{tabular}

\begin{tabular}{|c|c|c|}
\hline \multicolumn{3}{|c|}{ Horizontal Location of $P Q$} \\
\hline \multirow{2}{*}{$\begin{array}{l}\text { viscous state } \\
\qquad\left(\delta \rightarrow 90^{\circ}\right)\end{array}$} & $\begin{array}{l}\text { change due to } \\
\text { increase in } \eta_{0} / E_{\infty}\end{array}$ & $\begin{array}{c}\text { change due to } \\
\text { decrease in } \eta_{0} / E_{\infty}\end{array}$ \\
\hline & $\longleftarrow$ & $\longrightarrow$ \\
\hline \multicolumn{3}{|c|}{ Length of $P Q$} \\
\hline \multirow{2}{*}{$\begin{array}{l}\text { rate of change, } \\
\text { elastic } \leftrightarrow \text { viscous } \\
\qquad\left(\delta ; 0^{\circ} \leftrightarrow 90^{\circ}\right)\end{array}$} & $\begin{array}{l}\text { rapid at specific } \\
\text { frequency }\left(\delta=45^{\circ}\right)\end{array}$ & $\begin{array}{l}\text { gradual over a } \\
\text { wide range of } \\
\text { frequency }\end{array}$ \\
\hline & short $\bar{\AA}$ & long $\bar{\uparrow}$ \\
\hline
\end{tabular}
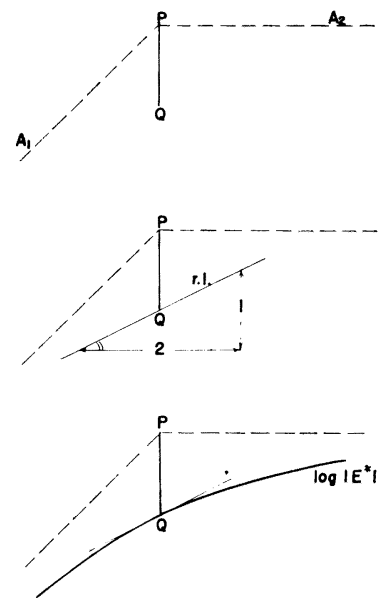

Fig. 15 Derivation of $\left|E^{*}\right|$ from characteristic line $P Q$.

$\left|E^{*}(i \omega)\right|$ curve from the characteristic line $P Q$. This procedure is as follows. When a characteristic line $P Q$ is given as shown in Fig. 15, two asymptotes $A_{1}$ and $A_{2}$ are drawn from the point $P$. $A_{2}$ is parallel to the abscissa and $A_{1}$ has a slope of $45^{\circ}$ to the abscissa. Since the $\left|E^{*}(i \omega)\right|$ curves pass the point $Q$ with the slope of $1 / 2$ (about $35^{\circ}$ ), we draw the reference line at $Q$ as shown in Fig. 15(b). $\left|E^{*}(i \omega)\right|$ curve can be drawn by starting from reference line at $Q$ and drawing smooth curve which approaches gradually $A_{1}$ and $A_{2}$ as shown in Fig. 15 (c).

Thus, we shall be able to estimate the viscoelastic properties of asphalt mixtures by the location and the length of the characteristic line $P Q$ plotted on the plane of $\log \left|E^{*}\right|$ and $\log \omega$.

\section{(5) Effects of Mixture Components on Viscoelastic Properties}

Characteristic lines at $15^{\circ} \mathrm{C}$ for all specimens tested are shown in Fig. 16(a), (b). In Fig. 16(a), the asphalt binder used had the Pen. 80/100 (P.I.=0.7) and in (b) the Pen. 20/40 (P.I. =+5).

It is interesting that the horizontal locations of characteristic lines in Fig. 16(a) are clearly apart from those in Fig. 16(b). Since the components of the materials were same for both cases except for asphalt binder used, it is apparent that the frequency characteristics of the mixtures were governed mainly by the asphalt properties (eg., Pen. and P.I.).

A characteristic line for asphalt binder itself is located at about $200 \mathrm{rad} . / \mathrm{sec}$. for the asphalt binder 20/40 as shown in Fig. 16(a), and for the binder $80 / 100$ it is at higher frequency than $300 \mathrm{rad} . / \mathrm{sec}$. so that it cannot be plotted in Fig. 16(b). The
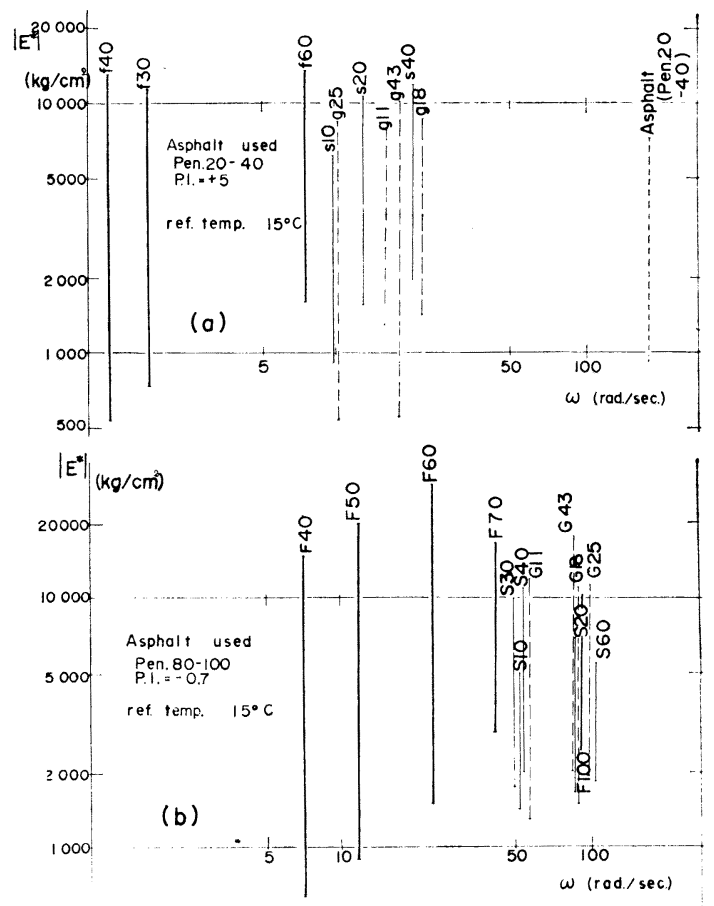

500

Fig. 16 Characteristic lines for all samples. 


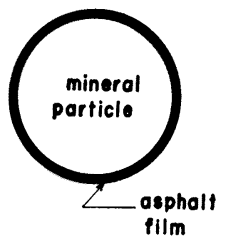

Fig. 17 Asphalt film covering on mineral particles.

differences between the characteristic lines for the asphalt itself and the mixtures show the mixing effects of filler and aggregates with pure asphalt. From Fig. 16 (a), (b), we can see that the location of characteristic line of asphalt binder itself are shifted towards left side by mixing mineral particles, especially for the filler particles. It can be concluded that the viscous properties of binder becomes less significant by the mixing of mineral particles even in the range of low frequencies.

The length of the characteristic lines for sandasphalt or gravel-asphalt mixture is not quite different from each other. However, the length for filler-asphalt mixture is much longer than those of other types of mixtures, but approaches that of others when the asphalt content is increased. We found that this difference in length of characteristic line depends on the difference in thickness of asphalt films. If we assume the asphalt film to be uniform as shown in Fig. 17 and the shape of the mineral materials to be spherical then the film can be obtained by the following equation

$$
t=\frac{d}{6} \frac{V_{A S}}{V_{F}+V_{S}+V_{G}}
$$

where

$t=$ film thickness $(\mathrm{mm})$,

$d=$ average diameter of aggregate $(\mathrm{mm})$,

$V_{A S} /\left(V_{F}+V_{S}+V_{G}\right)=$ asphalt content in volume.

The relations between the film thickness and the length of the characteristic lines are shown in Fig. 18 which shows that mixtures may be divided into two groups, one is the group whose film thickness exceeds about 5 micron and the other is the group whose film thickness is less than about 5 micron. Since the length of the characteristic line is independent of temperature and frequency, we may conclude that mechanical properies of thinner asphalt film less than about 5 micron should be different from those of thicker film. The same tendency was observed by other static tests in our laboratory.

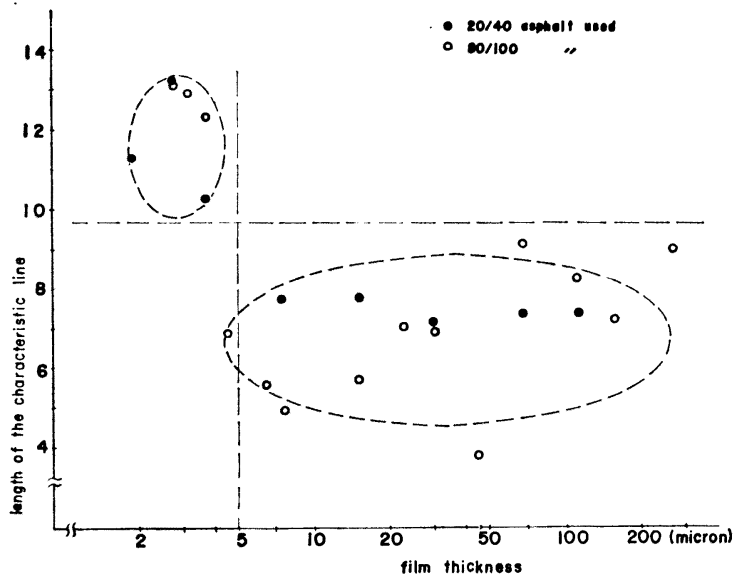

Fig. 18 Relations between the film thickness and the length of the characteristic lines.

\section{CONCLUSIONS}

The main purpose of this study was to obtain new indices which describe the mechanical properties of asphalt mixtures. Our conclusions are listed as follows:

(1) Time and temperature showed an approximately equivalent effect on the viscoelastic response of asphalt mixtures, so that the timetemperature superposition concept was valid to these materials to a satisfactory degree of accuracy.

(2) The relationship between shift factor $a_{T}$ and temperature proposed by Williams, Landel and Ferry was not satisfied for every asphalt mixture tested.

(3) The vector locus of complex elastic modulus $E^{*}(i \omega)$ for asphalt mixture was different from those of such simple models as Voigt type and Maxwell type.

(4) A characteristic line developed in this paper was considered to be a reasonable index in the analysis of mechanical properties of asphalt mixtures. It had the clear physical meanings such as a relaxation (or a retardation) spectrum, but is much easier to deal with. The forms of this representation is based upon the fact that the dynamic mechanical properties of asphalt mixtures change gradually from viscous to elastic state with the increase in frequency.

\section{ACKNOWLEDGEMENTS}

The authors would like to express their sincere appreciation to Mr. T. Iizuka, M. Nakamura and K. Suzuki, who were students of Tokyo Institute of Technology, for the cooperation in this study. 


\section{REFERENCES}

1) C. van der Poel: "A general system describing the visco-elastic properties of bitumens and its relation to test data", J. Appl. Chem. 4 (1954) 221.

2) Heukelom, W. and Klomp, G.: "Road design and dynamic loading”, Proc. A.A.P.T. vol. 33 (1964) 108-112.

3) Williams, M.L. Landel, R.F. and Ferry, J.D.: J. Amer. Chem. Soc., 77 (1955) 3701.

4) Krokosky, E. M. and Chen, J. P.: "Visco. elastic analysis of the Marshall test", M.I.T. Civil Eng. Dept. Rept. R63-41 (Nov. 1963). 